\title{
Quantum benchmark for storage and transmission of coherent states
}

\author{
K. Hammerer ${ }^{1}$, M.M. Wolf ${ }^{1}$, E.S. Polzik ${ }^{2,3}$, J.I. Cirac ${ }^{1}$ \\ ${ }^{1}$ Max-Planck-Institut für Quantenoptik, D-85748 Garching, Germany \\ ${ }^{2}$ QUANTOP, Danish Research Foundation Center for Quantum Optics \\ ${ }^{3}$ Niels Bohr Institute, DK 2100 Copenhagen, Denmark
}

\begin{abstract}
We consider the storage and transmission of a Gaussian distributed set of coherent states of continuous variable systems. We prove a limit on the average fidelity achievable when the states are transmitted or stored by a classical channel, i.e., a measure and repreparation scheme which sends or stores classical information only. The obtained bound is tight and serves as a benchmark which has to be surpassed by quantum channels in order to outperform any classical strategy. The success in experimental demonstrations of quantum memories as well as quantum teleportation has to be judged on this footing.
\end{abstract}

PACS numbers: 03.67.-a

Coherent distribution, storage and manipulation of quantum states is a technical challenge which received extensive theoretical and experimental interest in the last years stimulated by the promises of quantum information science [1]. A wide class of schemes can be very generally understood as an attempt to establish a channel for the reliable transmission of quantum states. This applies in particular to quantum teleportation [2, 3, 4, 5, 6, 7, 8, 9], where states are sent through an entanglement assisted classical channel, but just as well to the concept of a quantum memory 10, 11, 12, 13, 14, 15, 16, 17], where the channel acts in time rather than in space and the accent is on the state transfer between light and atoms. Concerning the reliable transmission and storage of quantum states it is clear that in the ideal case a quantum channel will always surpass a classical channel, i.e. any strategy where the quantum state is measured, the corresponding classical data stored or transmitted and then used to reconstruct the initial state as good as possible [18]. However, under realistic conditions a quantum channel suffers inevitably from imperfections such that it might become possible to achieve the same effect by means of a classical channel. Therefore there is need for a criterion which allows to distinguish an imperfect quantum channel from a perfect classical channel and justifies proclamation of success in the experimental demonstration of quantum teleportation and quantum memories.

Such a criterion has been derived some time ago for channels acting on finite dimensional systems 19, 20, 21, 22 and found applications in seminal experiments on quantum teleportation with single photons 4] and ions [5]. For channels acting on infinite dimensional systems a corresponding criterion was conjectured some time ago in [6, 23]. Though a proof for this criterion was yet to be found the claim for successful teleportation was based exclusively on this ground in several, likewise seminal teleportation experiments using EPR-squeezed light 6, 7, 8, 9]. The same criterion was applied very recently to a quantum memory experiment [10] where coherent states of light were stored in the collective spin of atoms.
In this paper we solve this longstanding problem and provide a rigorous proof for the criterion conjectured in 6, 23. This puts the central claims of experiments $6,7,8,9,10]$ - to have demonstrated a quantum gain in the transmission or storage of coherent states - on logically firm grounds. We emphasize that only now the success of these key experiments is rigorously validated. Moreover, the present result gives a solution to the state estimation problem for coherent states and leads to a closed expression for the accessible fidelity introduced in 24].

The paper is organized as follows: We first characterize a general classical channel mathematically and state the optimization problem to be solved here. Then we present this solution and give an elementary and rigorous proof. We close by relating the result to other work.

The figure of merit in terms of which the quality of a channel is quantified is usually taken to be the average fidelity achieved when the channel acts on a predefined set of input states [25]. Let $\left\{\left|\psi_{x}\right\rangle\right\}$ be this set and let an input $\left|\psi_{x}\right\rangle$ occur with a probability $p(x)$. If the channels's output is $E\left(\left|\psi_{x}\right\rangle\right)$ then the average fidelity is defined as

$$
\bar{F}=\sum_{x} p(x)\left\langle\psi_{x}\right| E\left(\left|\psi_{x}\right\rangle\right)\left|\psi_{x}\right\rangle
$$

This number is equal to one only for the ideal channel transmitting or storing every state perfectly. Now the task is to find the maximal value of $\bar{F}$ achievable with a classical channel, i.e. to identify the optimal measureand-prepare strategy. Any channel yielding a higher average fidelity is then necessarily quantum in the sense that it outperforms every strategy which is based on the mere storage or transmission of classical information. Any classical channel can be described by a POVM [1] measurement $\left\{M_{y}\right\}$ where $y$ denotes the outcome occurring with a probability $\left|\left\langle\psi_{x}\left|M_{y}\right| \psi_{x}\right\rangle\right|^{2}$ and a reconstruction rule $y \rightarrow \rho_{y}$ determining which state $\rho_{y}$ is prepared when $y$ was the measurement outcome. The channel then 
acts as

$$
E\left(\left|\psi_{x}\right\rangle\right)=\sum_{y}\left|\left\langle\psi_{x}\left|M_{y}\right| \psi_{x}\right\rangle\right|^{2} \rho_{y}
$$

The fidelity bound for classical channels relative to the set of input states $\left\{\left|\psi_{x}\right\rangle\right\}$ is then

$$
F_{\max }=\left.\sup _{M_{y}} \sup _{\rho_{y}} \sum_{x} \sum_{y} p(x)\left\langle\psi_{x}\left|M_{y}\right| \psi_{x}\right\rangle\right|^{2}\left\langle\psi_{x}\left|\rho_{y}\right| \psi_{x}\right\rangle .
$$

This optimization problem is known under the title state estimation in the theory of quantum detection and has in fact a long history [26, 27, 28, 29]. From the plethora of results known in this field the one concerning channels acting on $\mathbb{C}^{2}$ and an input set consisting of all pure states with a uniform distribution over the Bloch sphere received particular practical relevance in the last years. In [20] it was shown that for this case $F_{\max }=2 / 3$. This value was the appropriate benchmark in several teleportation experiments using single photons [4] and recently also trapped ions [5] and was beaten by measured fidelities ranging from .70 to .89 in [4] and from .75 to .78 in [5] proving the presence and necessity of entanglement in these experiments.

Less is known for channels acting on an infinite dimensional Hilbert space. Despite the increasing importance of coherent states $\{|\alpha\rangle\}$ for quantum communication and in particular quantum cryptography by now no classical-quantum bound has been proven for channels acting on these states. In 23] it was shown that if the coherent states are distributed in phase space according to a Gaussian distribution $p(\alpha)=\lambda / \pi \exp \left(-\lambda|\alpha|^{2}\right)$ an average fidelity $\bar{F}=(1+\lambda) /(2+\lambda)$ can be achieved by means of a heterodyne measurement, described by a POVM $\{|\alpha\rangle\langle\alpha| / \pi\}$, and the preparation of appropriate coherent states. It was conjectured there that this might be optimal but since then this question remained open. In fact, in the state estimation problem with minimum mean square error the heterodyne measurement turned out to be optimal 26]. However, this problem is different from the present one with respect to the figure of merit and due to the fact that in [26] the reconstruction of the state, which is crucial in our context, is not considered. Nevertheless, the value of $1 / 2$ attained for the flat distribution $(\lambda \rightarrow 0)$ was used as a criterion to verify teleportation in experiments [6, 7, 8, [9] using EPR-squeezed light where measured average fidelities range from .58 to .64 .

In the following we will settle this question by proving that for any classical strategy

$$
F_{\max } \leq \frac{1+\lambda}{2+\lambda}
$$

holds necessarily. Moreover, this bound is tight since it can be achieved by means of the strategy derived in [23], and thus equality holds in (2). This is the main result of this paper.

The proof we are going to present now is elementary and we start by simplifying and conveniently reformulating the problem. The first simplification relies on the fact that without loss of generality we can restrict the optimization in equation (11) to POVMs consisting of projectors $M_{y}=\left|\phi_{y}\right\rangle\left\langle\phi_{y}\right|\left(\left|\phi_{y}\right\rangle\right.$ not necessarily normalized) and also to pure states $\rho_{y}=\left|\chi_{y}\right\rangle\left\langle\chi_{y}\right|$. This is easily seen by noting that we can always decompose the POVM elements $M_{y}=\sum_{v}\left|m_{v, y}\right\rangle\left\langle m_{v, y}\right|$ and similarly the states $\rho_{y}=\sum_{w} q_{w, y}\left|r_{w, y}\right\rangle\left\langle r_{w, y}\right|$ such that we can write the average fidelity as

$$
\bar{F}=\sum_{x} \sum_{y, v, w} p(x)\left|\left\langle\psi_{x}\left|\sqrt{q_{w, y}}\right| m_{v, y}\right\rangle\right|^{2}\left|\left\langle\psi_{x} \mid r_{w, y}\right\rangle\right|^{2} .
$$

Absorbing the redundant parameters $v, w$ into $y$ and identifying $\sqrt{q_{w, y}}\left|m_{v, y}\right\rangle$ and $\left|r_{w, y}\right\rangle$ with $\left|\phi_{y}\right\rangle$ and $\left|\chi_{y}\right\rangle$ respectively we see that for any POVM there exists always another one which has the desired properties and yields the same average fidelity.

We therefore have for coherent input states $\{|\alpha\rangle\}$ with a Gaussian distribution $p(\alpha)=\lambda / \pi \exp \left(-\lambda|\alpha|^{2}\right)$

$$
\bar{F}=\sum_{y} \int d \alpha p(\alpha)\left|\left\langle\alpha \mid \phi_{y}\right\rangle\right|^{2}\left|\left\langle\alpha \mid \chi_{y}\right\rangle\right|^{2} .
$$

Note that the sum over $y$ stands symbolically for sums or integrations over a suitable measurable set. Using this expression for $\bar{F}$ and defining

$$
A_{\phi_{y}}=\int \mathrm{d} \alpha p(\alpha)\left|\left\langle\alpha \mid \phi_{y}\right\rangle\right|^{2}|\alpha\rangle\langle\alpha| .
$$

equation (1) can be reformulated more compactly as

$$
F_{\max }=\sup _{\left|\phi_{y}\right\rangle\left|\chi_{y}\right\rangle} \sum_{y}\left\langle\chi_{y}\left|A_{\phi_{y}}\right| \chi_{y}\right\rangle=\sup _{\left|\phi_{y}\right\rangle} \sum_{y}\left\|A_{\phi_{y}}\right\|_{\infty} .
$$

The optimization with respect to the reconstructed states $\left|\chi_{y}\right\rangle$ is trivial and implicitly performed in the last identity by noting that it is clearly best to prepare the state corresponding to the largest eigenvalue of $A_{\phi_{y}}$ for a given measurement outcome $y[36]$.

We proceed by proving a statement which is even stronger than (2) namely that

$$
\left\|A_{\phi}\right\|_{p} \leq \frac{1+\lambda}{\left[(2+\lambda)^{p}-1\right]^{1 / p}}\left\|A_{\phi}\right\|_{1}
$$

holds for all states $|\phi\rangle$ and all $p$-norms $\|A\|_{p}=\left[\operatorname{tr}\left\{A^{p}\right\}\right]^{1 / p}$. The main statement, equation (2), is deduced from equations (3) and (4) by taking the limiting case $p \rightarrow \infty$ of equation (4) in combination with the POVM property $\sum_{y}\left|\phi_{y}\right\rangle\left\langle\phi_{y}\right|=\mathbf{1}$, which in turn implies $\sum_{y}\left\|A_{\phi_{y}}\right\|_{1}=1$.

In order to prove inequalities (4) we exploit a trick which was already utilized in the context of additivity of 
output purities of bosonic channels in [30]. The properties of the trace allow us to write

$$
\begin{aligned}
\left\|A_{\phi}\right\|_{p}^{p}=\operatorname{tr}\left\{A_{\phi}^{p}\right\}= & \iint \mathrm{d} \alpha_{1} \cdots \mathrm{d} \alpha_{p} p\left(\alpha_{1}\right) \cdots p\left(\alpha_{p}\right) \\
& \times\left|\left\langle\phi \mid \alpha_{1}\right\rangle\right|^{2} \cdots\left|\left\langle\phi \mid \alpha_{p}\right\rangle\right|^{2} \\
& \times \operatorname{tr}\left\{\left|\alpha_{1}\right\rangle\left\langle\alpha_{1} \mid \alpha_{2}\right\rangle \cdots\left\langle\alpha_{p-1} \mid \alpha_{p}\right\rangle\left\langle\alpha_{p}\right|\right\} \\
= & \operatorname{tr}\left\{|\phi\rangle\left\langle\left.\phi\right|^{\otimes p} B\right\},\right. \\
\left\|A_{\phi}\right\|_{1}^{p}=\operatorname{tr}\left\{A_{\phi}\right\}^{p}= & \operatorname{tr}\left\{|\phi\rangle\left\langle\left.\phi\right|^{\otimes p} C\right\}\right.
\end{aligned}
$$

where we defined

$$
\begin{aligned}
B= & \iint \mathrm{d} \alpha_{1} \cdots \mathrm{d} \alpha_{p} p\left(\alpha_{1}\right) \cdots p\left(\alpha_{p}\right)\left\langle\alpha_{1} \mid \alpha_{2}\right\rangle \cdots\left\langle\alpha_{p} \mid \alpha_{1}\right\rangle \\
& \times\left|\alpha_{1}\right\rangle\left\langle\alpha_{1}|\otimes \cdots \otimes| \alpha_{p}\right\rangle\left\langle\alpha_{p}\right|, \\
C= & \bigotimes_{i=1}^{p} \int \mathrm{d} \alpha_{i} p\left(\alpha_{i}\right)\left|\alpha_{i}\right\rangle\left\langle\alpha_{i}\right| .
\end{aligned}
$$

These two operators commute evidently and thus can be diagonalized in the same basis. The diagonalization can be accomplished following the methods of [30]. One finds that both $B$ and $C$ can be expressed as a tensor product of (unnormalized) thermal states in certain Fourier modes attained from the original $p$ modes by a unitary transformation. Both operators are diagonal in the Fock basis corresponding to these new modes and are explicitly given by

$$
\begin{aligned}
& B=\frac{\lambda^{p}}{(2+\lambda)^{p}-1} \bigotimes_{i=1}^{p} \sum_{n_{i}=0}^{\infty}\left(\frac{1}{2+\lambda-d_{i}}\right)^{n_{i}}\left|n_{i}\right\rangle\left\langle n_{i}\right|, \\
& C=\left(\frac{\lambda}{1+\lambda}\right)^{p} \bigotimes_{i=1}^{p} \sum_{n_{i}=0}^{\infty}\left(\frac{1}{1+\lambda}\right)^{n_{i}}\left|n_{i}\right\rangle\left\langle n_{i}\right|
\end{aligned}
$$

where $d_{i} \in \mathbb{C}$ are the eigenvalues of a unitary matrix such that $\left|d_{i}\right|=1$. The exact values can easily be calculated but are of no relevance here.

Finally, let a product state $|\phi\rangle^{\otimes p}$ have an expansion in terms of Fock states given by $|\phi\rangle^{\otimes p}=\sum_{n_{1}, \ldots, n_{p}} c_{n_{1}, \ldots, n_{p}}\left|n_{1}, \ldots, n_{p}\right\rangle . \quad$ By construction we know that $0 \leq \operatorname{tr}\left\{|\phi\rangle\left\langle\left.\phi\right|^{\otimes p} B\right\}\right.$ and therefore

$$
\begin{aligned}
& \operatorname{tr}\left\{|\phi\rangle\left\langle\left.\phi\right|^{\otimes p} B\right\}=\right. \\
& =\left.\frac{\lambda^{p}}{(2+\lambda)^{p}-1}\left|\sum_{n_{1}, \ldots, n_{p}=0}^{\infty} \prod_{i=1}^{p}\left(\frac{1}{2+\lambda-d_{i}}\right)^{n_{i}}\right| c_{n_{1}, \ldots, n_{p}}\right|^{2} \mid \\
& \leq \frac{\lambda^{p}}{(2+\lambda)^{p}-1} \sum_{n_{1}, \ldots, n_{p}=0}^{\infty} \prod_{i=1}^{p}\left|\frac{1}{2+\lambda-d_{i}}\right|^{n_{i}}\left|c_{n_{1}, \ldots, n_{p}}\right|^{2} \\
& \leq \frac{\lambda^{p}}{(2+\lambda)^{p}-1} \sum_{n_{1}, \ldots, n_{p}=0}^{\infty} \prod_{i=1}^{p}\left(\frac{1}{1+\lambda}\right)^{n_{i}}\left|c_{n_{1}, \ldots, n_{p}}\right|^{2} \\
& =\frac{(1+\lambda)^{p}}{(2+\lambda)^{p}-1} \operatorname{tr}\left\{|\phi\rangle\left\langle\left.\phi\right|^{\otimes p} C\right\} .\right.
\end{aligned}
$$

Taking the $p^{\text {th }}$ root of this sequence yields directly relation (4) and completes the proof.
The result assures that (in the case of a flat distribution) the fidelity limit of $1 / 2$ is in fact appropriate in comparing quantum channels for coherent states of continuous variables with an optimal classical channel, justifying its application as a benchmark in continuous variable teleportation [6, 7, 8, 9] ex post and in future experiments testing the performance of continuous variable quantum memories [10, 11, 12, 13. In particular, in a recent experimental demonstration of the quantum state transfer from light onto atoms [10] the bound (2) has been used to demonstrate that the quantum memory has indeed exceeded the classical limit of the measureand-prepare strategy. The present proof provides firm grounds for such a statement.

We note that a measure-and-prepare scheme can be considered as a 1-to- $\infty$ cloning machine, when we just duplicate the preparation device. In fact, in this context for the special case of a flat distribution $(\lambda \rightarrow 0)$ an independent proof based on the covariance of the problem is given in [31].

The criterion derived here allows to test wether a given channel yields a higher quality of storage or transmission (measured in terms of the average fidelity) than what is possible by classical means. We would like to point out that there exist other criteria in the literature 31, 32, 33, 34 allowing to test different requirements. In particular if a channel has to be secure (in the sense that its action excludes the existence of a clone of the input state holding a higher fidelity than the channel's output) it has to outperform the best 1-to-2 cloning machine, which is more demanding than what was considered here 33]. For channels acting on the set of coherent states with a flat distribution this was analyzed in 31, 32, 33]. As shown in 32, 33] the best Gaussian 1-to-2 cloning machine yields a fidelity benchmark of $2 / 3$ while the optimal non-Gaussian strategy yields a value of $\approx 0.6826$ as was derived in 31].

Finally, we would like to point out that an experimental demonstration of a fidelity larger than $1 / 2$ does not disprove the existence of a classical model in the sense of a local hidden variable theory able to describe the physical process 34, 35. When we claim that it does prove the non-classicality or quantumness of the respective channel then this has to be understood in the sense that no classical measure-and-prepare strategy can give the same result within the framework of quantum mechanics.

In conclusion, we presented and proved a tight upper bound on the average fidelity achievable by a classical channel for coherent states of continuous variables subject to a Gaussian distribution over the phase space. This limit has to be surpassed by a quantum channel in order to outperform any competing classical strategy and is thus of direct experimental relevance in quantum teleportation of and quantum memories for continuous variables. The presented result in particular validate the outstanding experimental achievements in storing and teleport- 
ing continuous variable quantum states. The techniques, which led to the proof of the bound, in principle apply also to other sets of states in both continuous variable and finite dimensional systems. Depending on the considered sets and distributions they might thus yield similar quantum benchmarks in other contexts.

M.M.W. thanks Reinhard F. Werner and Ole Krüger for valuable discussions. This work was supported by the EU project COVAQIAL, the Danish National Research Foundation and the Kompetenznetzwerk Quanteninformationsverarbeitung der Bayerischen Staatsregierung.

[1] M.A. Nielsen, I.L. Chuang, Quantum Computation and Qauntum Information, Cambridge University Press (2000), Cambridge

[2] C.H. Bennett et al., Phys. Rev. Lett. 70, 1895 (1993)

[3] L. Vaidman, Phys. Rev. A, 49, 1473 (1994), S.L. Braunstein, H.J. Kimble, Phys. Rev. Lett., 80, 869 (1998)

[4] D. Bouwmeester et al., Nature 390, 575 (1997), D. Fattal et al., Phys. Rev. Lett. 92, 037904 (2004), I. Marcikic et al., Nature 421, 509 (2003), Y.-H. Kim et al., Phys. Rev. Lett. 86, 1370 (2001), D. Boschi et al., Phys. Rev. Lett. 80, 1121 (1998), R. Ursinn et al., Nature 430, 849 (2004), J.-W. Pan et al., Phys. Rev. Lett. 86, 4435 (2001) [The average fidelities in these experiments were $.70, .80, .81, .83, .85, .87$ and .89 respectively.]

[5] M.Riebe et al., Nature 429, 734 (2004), M.D. Barrett et al., Nature 429, 737 (2004) [The average fidelities in these experiments were .75 and .78 respectively.]

[6] A. Furusawa, S.L. Braunstein, C.A. Fuchs, H.J. Kimble, E.S. Polzik, Science 282, 706 (1998) [The average fidelity in this experiment was .58]

[7] T.C. Zhang, K.W. Goh, C.W. Chou, P. Lodahl, K.J. Kimble, Phys. Rev. A 67, 033802 (2003) [The average fidelity in this experiment was .61]

[8] W.P. Bowen, N. Treps, B.C. Buchler, R. Schnabel, T.C. Ralph, H.A. Bachor, T. Symul, P.K. Lam, Phys. Rev. A 67, 032302 (2003) [The average fidelity in this experiment was .64]

[9] H. Yonezawa, T. Aoki, A. Furusawa, Nature 431, 430 (2004) [The average fidelity in this experiment was .63]

[10] B. Julsgaard, J. Sherson, J.I. Cirac, J. Fiurek, E.S. Polzik, Nature 432, 482 (2004)

[11] A. Kuzmich et al., Phys. Rev. Lett. 79, 4782 (1997), A. E. Kozhekin et al., Phys. Rev. A 62, 033809 (2000)

[12] C. Schori et al., Phys. Rev. Lett. 89, 057903 (2002), B. Julsgaard et al., Quant. Inform. and Comp., spec. issue 3, 518 (2003)
[13] A. Dantan, M. Pinard, Phys. Rev. A 69043810 (2004), A. Dantan, A. Bramati, M. Pinard, Europhys. Lett. 67, 881 (2004)

[14] M. Fleischhauer, M.D. Lukin Phys. Rev. Lett 84, 5094 (2000), M. Fleischhauer, M.D. Lukin, Phys. Rev. A 65, 022314 (2002)

[15] C. Liu et al., Nature 409, 490 (2001)

[16] M. Bajcsy et al., Nature 426, 638 (2003)

[17] J.I. Cirac et al., Phys. Rev. Lett. 78, 3221 (1997)

[18] This is a direct consequence of the no-cloning theorem. D. Dieks, Phys. Lett. A 92, 271 (1982), W.K. Wootters, W.H. Zurek, Nature 299, 802 (1982), see also R.F. Werner, Quantum Information and Quantum Computing (Lecture Notes), http://www.imaph.tu-bs.de/qi/qi.html

[19] S. Popescu, Phys. Rev. Lett. 72, 797 (1994)

[20] S. Massar, S. Popescu, Phys. Rev. Lett. 74, 1259 (1995)

[21] R. Derka et al., Phys. Rev. Lett. 80, 1571 (1998)

[22] D. Bruss et al., Phys. Rev. Lett. 81, 2598 (1998)

[23] S.L. Braunstein et. al., J. Mod. Opt. 47, 267 (2000) [Note in particular the footnote on p. 275]

[24] K.M.R. Audenaert, C.A. Fuchs, C. King, A. Winter, Quantum Inf. Comput. 4(1), 1 (2004)

[25] In the context of continuous variable teleportation figure of merits different from the fidelity have been proposed in order to evaluate non unit gain teleportation. See W.P. Bowen, N. Treps, B.C. Buchler, R. Schnabel, T.C. Ralph, T. Symul, P.K. Lam, IEEE J. Sel. Top. Quant. El. 9, 1519 (2003)

[26] H.P. Yuen, M. Lax, IEEE Trans. Inform. Theory 19, 740 (1973)

[27] A.S. Holevo, Probabilistic and Statistical Aspects of Quantum Theory (North-Holland, Amsterdam, 1982)

[28] C.W. Helstrom, Quantum Detection and Estimation Theory (Academic, New York, 1976)

[29] H.P. Yuen et al., IEEE Trans. Inform. Theory 21, 125 (1975)

[30] V. Giovannetti, S. Lloyd, L. Maccone, J.H. Shapiro, B.J. Yen, quant-ph/0404037

[31] N.J. Cerf, O. Krueger, P. Navez, R.F. Werner, M.M. Wolf, quant-ph/0410058

[32] N.J. Cerf, A. Ipe, and X. Rottenberg, PRL 85, 1754 (2000)

[33] F. Grosshans, P. Grangier, Phys. Rev. A 64, 010301 (2001)

[34] C.M. Caves, K. Wodewicz, Phys. Rev. Lett 93, 040506 (2004)

[35] S.L. Braunstein, Phys. Rev. A 64, 022321 (2001)

[36] The norm used in equations (3) and (4) is a special case of a $p$-norm defined by $\|A\|_{p}=\operatorname{tr}\left\{A^{p}\right\}^{1 / p}$. $\|A\|_{\infty}$ is just the largest eigenvalue of $A$ and $\|A\|_{1}=\operatorname{tr}\{A\}$. For all $p^{\prime} \geq p$ it holds that $\|A\|_{p^{\prime}} \leq\|A\|_{p}$. 